\title{
Pediatric Sedation Outside of the Operating Room: A Multispecialty International Collaboration
}

\author{
Keira P. Mason (Editor). Springer Science + Business Media: New York, USA 2012. \\ \$99.00, 518 pages, ISBN 978-0-387-09713-8
}

\author{
Amy Roeske, MD
}

Received: 16 March 2012/ Accepted: 23 March 2012/Published online: 19 April 2012

(C) Canadian Anesthesiologists' Society 2012

This recently published textbook is a unique international collaboration of specialists on the topic of pediatric sedation. The contributors are mainly from the United States; however, there are also contributions from five international authors (Netherlands (2), New Zealand, Africa, and United Kingdom). Furthermore, most of the authors are anesthesiologists, although specialists representing the fields of emergency medicine, pediatrics, intensive care, dentistry, gastroenterology, and food and drug administration are also included in the text.

The editor of this textbook is a leading expert on the subject of pediatric sedation. She has numerous published articles and is the main author of the chapter on this topic in two well-known pediatric anesthesia textbooks, A Practice of Anesthesia for Infants and Children, $4^{\text {th }}$ edition by Charles J. Cote et al. and Smith's Anesthesia for Infants and Children, $7^{\text {th }}$ edition by Etsuro Motoyama and Peter Davis.

This textbook is geared not only towards anesthesiology but also towards all specialties that provide sedation to pediatric patients. The initial chapters in Part 1 of the text, "Pediatric Sedation Outside the Operating Room", comprise a brief review of the history of sedation, a very basic overview of sedation, and a comparison of sedation scales. In one chapter, there is a review and comparison of some of the most recent American sedation guidelines, including guidelines from the American Academy of Pediatrics (AAP), the American Society of Anesthesiologists (ASA), and the American College of Emergency Physicians. Of note, there is an error in the ASA/AAP fasting guidelines; on page 23 , they neglect to specify timing of fasting for

\footnotetext{
A. Roeske, MD ( $\square)$

Children's Hospital of Eastern Ontario (CHEO),

Ottawa, ON, Canada

e-mail: aeroeske@gmail.com
}

formula or nonhuman milk and they do not specify eight hours of fasting for full meals. International guidelines (United Kingdom, Netherlands, and South Africa) are discussed later on in the book. After the discussion of American sedation guidelines, there is a basic review of pediatric anatomy, physiology, pharmacology, and monitoring. Surprisingly, despite a detailed description for the many sedative drugs reviewed in the pharmacology chapter, there is no discussion regarding dosing, though dosing is discussed in multiple instances in other chapters. Part 1 of the text finishes with a chapter on billing, which is very specific to the American system.

Part II, "Sedation Models Delivered by Different Specialties: A Global Voyage" includes chapters devoted to the role of hospitalists and anesthesiologists in a sedation service, followed by chapters describing sedation for different areas in the hospital, specifically, gastrointestinal procedures, intensive care unit, emergency room, and dental procedures. Each chapter has some repetition, particularly regarding medications; however, the authors provide their individual insights on each drug specific to their area of expertise. The final two chapters provide some insight into how sedation services are organized in Europe and Africa.

Part III, "Safety in Sedation" begins with a chapter describing how the majority of drugs used for pediatric sedation are not approved for use in children, and it includes an outline of the steps required to obtain Food and Drug Administration approval. There is an important discussion regarding adverse events, the importance of reporting adverse events to create benchmark data, and how to prevent a malpractice lawsuit related to these events. Part III ends with a discussion of strategies to ensure a safe sedation service.

Part IV, "Sedation into the Twenty-Second Century", includes a detailed discussion on target-controlled infusion 
systems, again with some repetition from the chapter on pharmacokinetics. This discussion is followed by a summary of complementary and alternative medicine options, including music, hypnotherapy, guided imagery, acupuncture, and sucrose. There is a discussion in the final chapter regarding questions that remain unanswered in the area of pediatric sedation.

This book is an excellent resource for any physician looking to develop or improve the sedation service at their institution. Some topics are geared more towards nonanesthesiologists performing sedation; however, it is interesting for anesthesiologists to learn about sedation practices in other specialties or other areas in the world. It is a good reference book to retain in a departmental library. A highlight in the book, which follows some of the chapters, is the use of clinical case studies with suggestions for management based on the author's personal experience. A recommendation for the future would be to include the online version of the book in the price of the print version, as the current trend is towards e-learning.

Competing interests None declared. 